\title{
IMPROVED HIERARCHIAL INTRA PREDICTION BASED ON ADAPTIVE INTERPOLATION FILTERING FOR LOSSLESS COMPRESSION
}

\author{
Li-Li Wang and Wan-Chi Siu \\ Department of Electronic and Information Engineering \\ The Hong Kong Polytechnic University \\ Hung Hom, Kowloon, Hong Kong
}

\begin{abstract}
Often the adaptive interpolation filter provides a more accurate interpolation technique in the formulation of the sub-pixel reference frame for motion estimation and compensation. Inspired by its possible advantages, we make use of the adaptive interpolation filter for efficient lossless intra prediction in H.264/AVC. Specifically, four subblocks are firstly formed by sampling pixels in one macroblock/block, and then a hierarchical intra prediction is performed on these subblocks. The first subblock is predicted based on the intra spatial prediction method in H.264/AVC, and subsequently it is encoded and reconstructed. The remaining three subblocks are then predicted based on adaptive interpolation filters by using the neighboring reconstructed samples. The residual block is encoded by rate optimization. Experimental results show that the proposed algorithm can improve the compression efficiency of lossless intra coding.
\end{abstract}

\section{INTRODUCTION}

Lossless coding technique has drawn an increasing attention recently. Besides its applications for image coding [1, 2], it also can be applied to video coding, such as using the H.264/AVC standard [3]. The lossless coding method used in H.264/AVC standard can be considered as a state-of-the-art representative for video coding standards. However, the encoding efficiency is still low since there is no transform and quantization operations in the lossless video coding. Another problem is that, since the H.264/AVC has a blockbased coding structure [3], samples away from the references are not predicted well due to poor correlation. In order to solve this problem, two algorithms based on samplewise DPCM instead of using a block-based approach are presented in [4] and [5]. In [4], the samplewise DPCM approach is firstly suggested for lossless intra prediction. For adopting near samples as the reference for prediction, the compression efficiency is enhanced. This algorithm has been adopted in the H.264/AVC standard. However, only the modes with one sample predictor can be improved by using the samplewise DPCM method. As a result, the performance is limited. In [5], the authors extended the sample DPCM concept to all intra prediction modes by using a simple linear interpolation. Hence, higher compression efficiency is obtained. In [6], we proposed a hierarchical intra prediction (HIP) algorithm making use of intensity gradient measurement to further improve the coding efficiency of lossless intra prediction in H.264/AVC.

Recently, adaptive interpolation filter has achieved a significant application in high efficiency video coding [7] for the formulation of a fractional-pixel reference frame in subpixel motion estimation and motion compensation. In this paper, we apply the adaptive interpolation filter to the hierarchical intra prediction to improve the lossless compression for videos. In this algorithm, pixels in a macroblock/block are firstly sampled into four subblocks. Intra prediction is then performed based on intra directional prediction or adaptive interpolation filter for each subblock. After the prediction has been finished, two coding modes combined with the rate optimization proposed by us in [6] are used to encode the residual block. Experimental results show that, the proposed algorithm can improve the compression efficiency for videos compared with other lossless intra coding methods in the literature.

\section{LOSSLESS INTRA CODING IN H.264/AVC}

For lossless intra prediction in the FRExt high profiles of H.264/AVC [3], four basic types of intra prediction for the luma component are available. They are 16x16 luma prediction (I16MB type), 8x8 luma prediction (I8MB type), $4 \times 4$ luma prediction (I4MB type) and IPCM [3], which are the same as those in the intra prediction types used in lossy coding process. There are totally 9 prediction modes in the I4MB/I8MB types, and four prediction modes in I16MB type. As for the intra prediction for two Chroma components, four modes are available for each $8 \times 8$ block. Details can be found in [3].

After the intra prediction is performed, the residual block is obtained. Different from the lossy video coding in H.264/AVC, the residual block is directly scanned and encoded into the bitstream without transformation or quantization in the process of lossless coding. Consequently, the intra lossless compression becomes not efficient. Moreover, we also note that the intra block/MB is predicted as a whole by an extrapolation of the neighboring reconstructed pixels in the original H.264/AVC standard. As a result, samples which are far from their references may not be predicted well due to poor correlation. Especially for the chroma blocks and luma blocks predicted by I8MB or I16MB type, the poor prediction is obvious since a larger block size is used.

\section{PROPOSED LOSSLESS INTRA COMPRESSION}

Recall that a filter with adaptive coefficients has achieved a good performance for the interpolation of a MB in [7] for high efficiency video coding. With this advantage as an inspiration, we propose a new lossless intra compression algorithm for videos. Details are shown as follows. 


\subsection{Hierarchical Block Pattern}

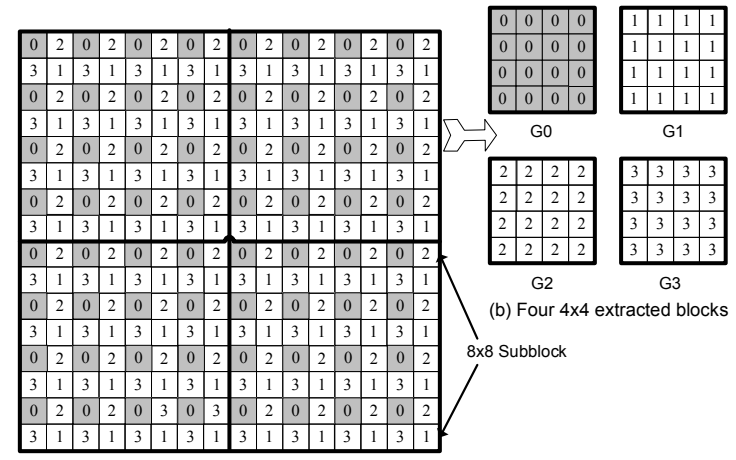

(a) The current Luma MB

Fig. 1 Structure of HIP for I8MB type

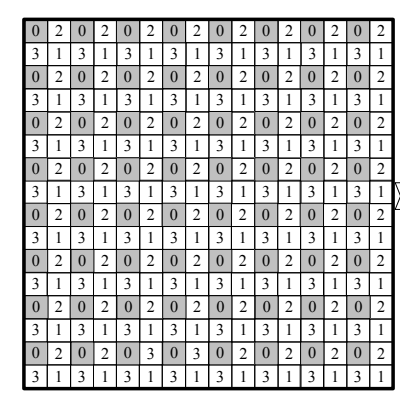

(a) The current Luma MB

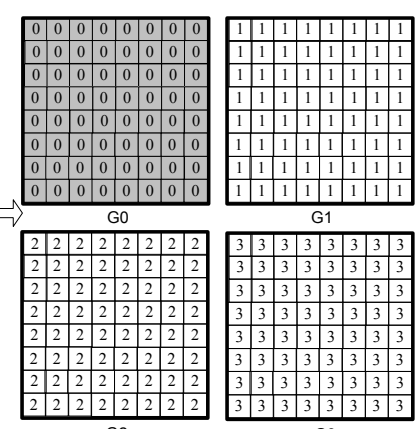

(b) Four $8 \times 8$ extracted blocks
Fig. 2 Structure of HIP for I16MB type

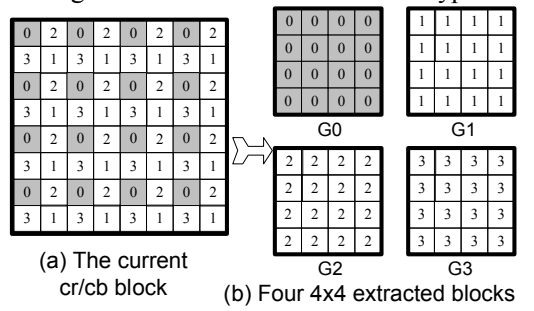

Fig.3 Structure of HIP for Chroma prediction

To improve the prediction accuracy of samples away from their references, we suggest that pixels in a $\mathrm{MB} /$ block be predicted hierarchically. Figs.1-3 show the structures of hierarchical intra prediction for the I8MB type, I16MB type and Chroma blocks, respectively. As shown in these figures, the pixels in each prediction unit specified in H.264/AVC, such as $8 \times 8$ for I8MB type/Chroma blocks and $16 \times 16$ for I16MB type are sampled into four subblocks, which are labeled as G0, G1, G2 and G3.

\subsection{Hierarchical intra prediction}

Note that samples in G0 in Figs.1-3 can be seen as a lowresolution block, which comes from a decimation/downsample result of the original block. In other words, the recovery/prediction of the original block is, thus, an inverse problem. Therefore, we refer to the samples labeled as 0 as reference, and aim at constructing the original block as its super resolution version. In order to construct the original block, namely, for predicting the samples labeled as 1,2 and 3 , a block-based two-dimensional adaptive interpolation filter is used. The predicted structures for pixels in different subblocks are specified as shown in Fig. 4.

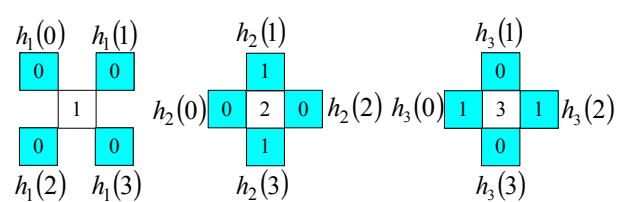

(a)

(b)

(c)

Fig.4 Predicted structures of samples in (a)G1, (b)G2, and (c)G3

Taking Fig.4 (a) as an example, the model for samples in $\mathrm{G} 1$ can be described as

$$
p_{i, j}^{1}=\sum_{m=0}^{1} \sum_{n=0}^{1} h_{1}(2 m+n) f_{i+m, j+n}^{0}
$$

where $\mathrm{i}, \mathrm{j}=0,1,2,3$ (same as below). Symbol $p_{i, j}^{1}$ denotes the predicted value of the sample in position $(i, j)$ of the extracted block $\mathrm{G} 1$, and $f_{x, y}^{0}$ denotes the pixel value in position (x, y) of the extracted block G0. In our implementation, four filter coefficients $\left\{h_{1}(k)\right.$, for $k=0,1,2$, 3 \} are used. Note that the filter length is set to 4 for sake of simplicity. In fact, it can be any other meaningful values. The proposed lossless intra coding algorithm is thus formulated as follows:

Step 1: Pixels in G0 are firstly predicted and reconstructed based on the intra prediction method in H.264/AVC. To improve the predicted accuracy, the DPCM concept in [5] is applied to each mode in the intra prediction of H.264/AVC. Since it is a lossless coding process, the reconstructed block $\mathrm{G} 0$ is the same as the original G0.

Step 2: The four filter coefficients, $\left\{h_{1}(m)\right.$, for $m=0,1,2$, $3\}$, are calculated for the samples in G1 by the minimisation of the prediction error energy. By using the obtained filter coefficients and the reference pixels in G0, the predicted value of each pixel in G1 is calculated based on (1).

In this step, the error of the prediction is calculated as

$$
e_{i, j}^{1}=f_{i, j}^{1}-p_{i, j}^{1}
$$

Note that the desired sample $f_{i, j}^{1}$ is not available in the decoder side. In order to avoid using the original pixel value during the process of energy minimization, the filter coefficients are estimated by using the geometric duality between the low-resolution covariance and the highresolution covariance based on the theory in [8]. Fig.5 shows the geometric duality between the high-resolution covariance and the low-resolution covariance for samples in the three extracted blocks.

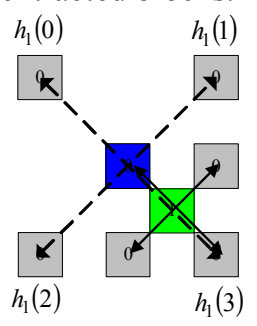

(a)

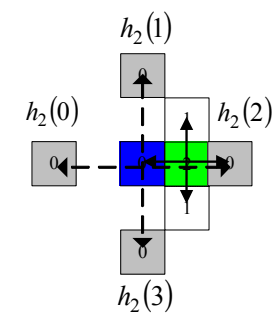

(b)

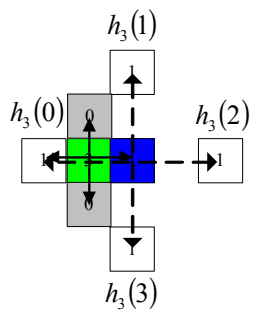

(c)
Fig. 5 Geometric duality for the interpolation of samples in (a) G1, (b) G2 and (c) G3

For example, in Fig.5 (a), the sample in blue color should be predicted instead of predicting the sample in green 
color based on geometric duality. The predicted error is given as follows:

$$
\begin{aligned}
& e_{i, j}^{0}=f_{i, j}^{0}-p_{i, j}^{0} \\
& p_{i, j}^{0}=\sum_{m=0}^{1} \sum_{n=0}^{1} h_{1}(2 m+n) f_{2(i+m), 2(j+n)}^{0}
\end{aligned}
$$

For each $4 \times 4 / 8 \times 8$ block, four filter coefficients have to be solved by minimizing the mean-square error. After calculation, the filter coefficients in matrix form can be represented as follows:

$$
\mathbf{H}_{c}^{o p t}=\left(\mathbf{R}_{f f}^{c}\right)^{-1} \mathbf{R}_{f d}^{c}
$$

In (5), $\mathbf{H}_{c}^{\text {opt }}, \mathbf{R}_{f f}^{c}$ and $\mathbf{R}_{\mathrm{fd}}$ are expressed as follows:

$$
\begin{aligned}
\mathbf{H}_{c}^{o p t} & =\left[\begin{array}{llll}
h_{c}^{o p t}(0) & h_{c}^{o p t}(1) & h_{c}^{o p t}(2) & h_{c}^{o p t}(3)
\end{array}\right]^{T} \\
\mathbf{R}_{f f}^{c} & =\mathbf{C}^{T} \mathbf{C} / N^{2} \\
\mathbf{R}_{f d}^{c} & =\mathbf{C}^{T} \mathbf{F} / N^{2}
\end{aligned}
$$

where $\mathrm{N}^{2}$ represents the number of pixels used in the training window. $\mathrm{N}$ is set to 4 and 8 for $4 \times 4$ and $8 \times 8$ blocks, respectively. $\mathbf{F}$ is a vector composed of the training data with $\mathrm{NxN}$ pixels inside the training subblock (i.e. $\mathrm{G} 0$ for $\left\{\mathrm{h}_{1}(\mathrm{~m})\right.$, for $\mathrm{m}=0,1,2,3\}$ ), and $\mathbf{C}$ is an $\mathrm{N}^{2} \mathrm{x} 4$ matrix whose $\mathrm{i}^{\text {th }}$ row consists of four predictors $\left\{s_{i j}^{c}\right.$, for $\left.\mathrm{j}=0,1,2,3\right\}$ as shown below and with reference to Fig. 5 for predicting the $i^{\text {th }}$ element in $\mathbf{F}$. When there are less than four predictors for some predicted samples in G0, the corresponding samples in G1 based on the geometric duality are predicted by using an intensity gradient measurement method proposed in [6].

$$
\mathbf{F}=\left[\begin{array}{c}
f_{0,0}^{0} \\
f_{1,0}^{0} \\
\vdots \\
f_{N-1, N-1}^{0}
\end{array}\right] \text {, and } \mathbf{C}=\left[\begin{array}{cccc}
s_{00}^{0} & s_{01}^{0} & s_{02}^{0} & s_{03}^{0} \\
s_{10}^{0} & s_{11}^{0} & s_{12}^{0} & s_{13}^{0} \\
\vdots & \vdots & \vdots & \vdots \\
s_{(N-1)^{2} 0}^{0} & s_{(N-1)^{2} 1}^{0} & s_{(N-1)^{2} 2}^{0} & s_{(N-1)^{2} 3}^{0}
\end{array}\right]
$$

Step 3: Similarity, the four filter coefficients $\left\{h_{2}(m)\right.$ and $\mathrm{h}_{3}(\mathrm{~m})$, for $\left.\mathrm{m}=0,1,2,3\right\}$ are calculated for the pixels in G2 and G3 based on geometric duality, respectively. The predicted value of each pixel in G2 and G3 is then calculated by using these filter coefficients and the reference pixels in G0 and G1 with reference to Fig.4. The residual block is then encoded into bitstream.

\subsection{Further improvement about the proposed algorithm}

Compared with the I8MB type, the I16MB type and the Chroma prediction, the I4MB type is associated with a smaller block. As a result, only the DPCM method in [5] is used to improve the prediction of I4MB type for our lossless prediction process.

Notice that some residual blocks show similar characteristics to that of the quantized DCT coefficients, and others do not. According to these two situations, two coding modes proposed in [6] as shown in Fig.6: one-layer coding (OLC) mode and two-layer coding (TLC) mode, are adopted to compress a $4 \times 4$ residual block. As shown in Fig.6, quantization (Q) is required in the TLC. Based on our experimental work on more than 30 video sequences, we found when the quantization parameter is set to 24 , the best compression efficiency of an encoder based on [9] can be achieved. Moreover, the rate optimization is used to select a better coding mode. The mode with a smaller rate cost is selected as the final coding mode for the residual block. Due to the introduction of two coding modes, a flag (0: one-layer coding mode; 1: two-layer coding mode) is required to indicate which mode is used for each $4 \times 4$ unit during the encoding process, and this flag is required to be written into the bit stream. To maximally increase entropy coding efficiency of these flags, an improved context-based adaptive variable length coding scheme (ICAVLC) proposed by us is used to encode the flags. Details can be found in [6].

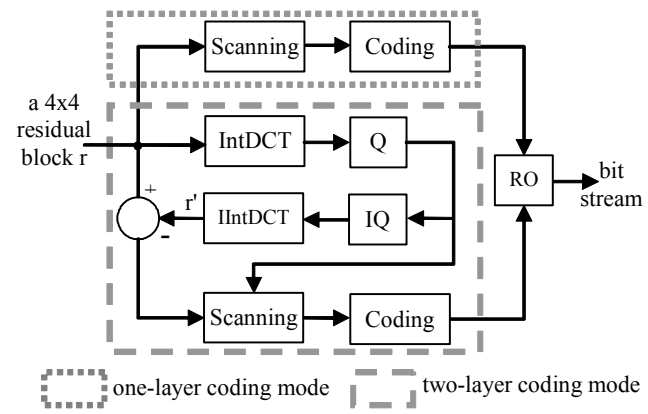

Fig. 6 Two coding modes for a $4 \times 4$ residual block

(IntDCT: Integer DCT; IIntDCT: Inverse Integer DCT; IQ:

Inverse Quantization; RO: Rate Optimization)

\section{EXPERIMENTAL RESULTS}

The proposed algorithm has been implemented on the platform of reference software JM 18.0 [9] to evaluate the performance. A large number of video sequences with YUV 4:2:0 format were used in this experiment. For each video sequence, 150 frames were encoded. The FRExt high profiles of H.264/AVC with specifications provided in [10] were set. To evaluate the efficiency of the lossless coding technique, only intra coded structure was performed.

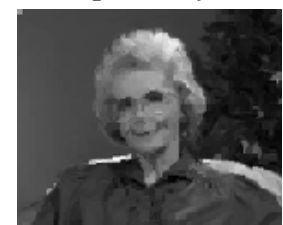

(a1) JM18.0 PF

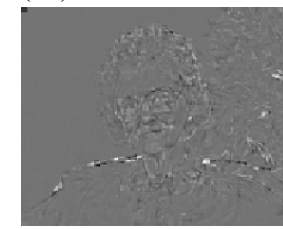

(a2) JM18.0 RF

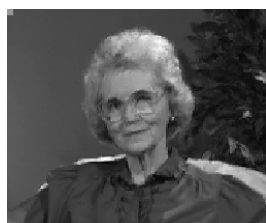

(b1) SIDPCM PF

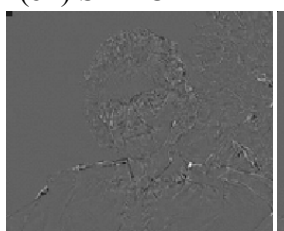

(b2) SIDPCM RF

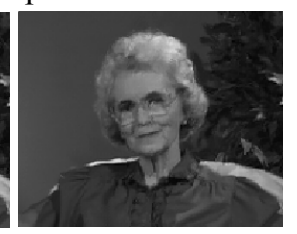

(c1) HIPAIF PF

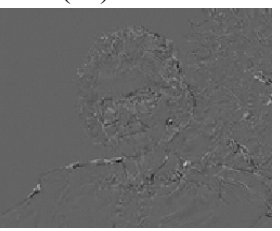

(c2) HIPAIF RF
Fig.7 Experimental results on Luma component for the first frame of Grandmother.QCIF sequence

Fig.7 gives an example about the predicted frames (PF) and their corresponding residual frames (RF) for several lossless coding algorithms. From Fig.7, we can see that the proposed algorithm by applying adaptive interpolation filtering technique to HIP (HIPAIF) makes the best prediction as shown in Fig.7 (c1) among all these algorithms, 
and thereby the information left in the residual frame (c2) is the smallest. The reason is that samples in G1, G2 and G3 are predicted using the block-based AIF and the neighboring reconstructed samples which are equal to the original samples in a lossless coding process, the correlation between them is generally higher. The AIF can learn the local structures adaptively. As a result, samples in G1, G2 and G3 can be well predicted.

Table I shows the experimental results for four lossless intra prediction algorithms in terms of the saving percentages of coding bits compared with the block-based method used in the original H.264/AVC standard (H.264_LS). From this table, we can see that the algorithm based on DPCM in [4] (H.264_DPCM) has shown a large saving percentage compared with H.264_LS. By contrast, the method using simple interpolation and DPCM (SIDPCM) in [5] makes a further improvement. Moreover, we can see from Table I that JPEG LS in [1] usually shows an improvement in the compression ratio compared with H.264_LS. Based on the experimental results in [4], we have found that JPEG_LS can achieve better performance than motion JPEG2000 in [2] for lossless compression of video sequences. However, when compared with our algorithm, it can be seen that our algorithm usually gives the best compression results for most of the tested video sequences. According to our experimental results, about an averaged $80 \%$ increase of encoding time compared with H.264/AVC is required due to the use of the adaptive interpolation filter and the rate optimization of two coding modes for residual blocks. However, it is less important for a high compression algorithm when it is heading for High Efficiency Video Coding (HEVC/H.265) [11]. Moreover, some fast rate estimation algorithms, such as the one in [12], can also be combined with this algorithm to reduce the encoding complexity.

Table I Percentages of saving bits for tested sequences

\begin{tabular}{|c|c|c|c|c|c|c|}
\hline \multirow{3}{*}{\multicolumn{2}{|c|}{ Sequence }} & \multirow{3}{*}{$\begin{array}{c}\text { Bits } \\
\text { H.264_LS } \\
\text { (H.264/AVC } \\
\text { Standard) }\end{array}$} & \multicolumn{4}{|c|}{ Saving Bits [\%] } \\
\hline & & & JPEG & H.264 & SIDPCM & HIPAIF \\
\hline & & & & & & \\
\hline \multirow{6}{*}{ QCIF } & Coastguard & 29279040 & -0.900 & 8.885 & 10.057 & 12.218 \\
\hline & Monitor & 26888775 & 13.550 & 8.330 & 10.568 & 15.506 \\
\hline & Foreman & 27832975 & 3.959 & 6.457 & 8.161 & 11.502 \\
\hline & Mobile & 40413815 & 13.143 & 3.587 & 4.725 & 11.563 \\
\hline & Carphone & 24726855 & 9.597 & 8.587 & 10.743 & 14.193 \\
\hline & Average & 29828292 & 7.870 & 7.169 & 8.851 & 12.996 \\
\hline \multirow{6}{*}{ CIF } & Container & 101859260 & 5.526 & "5.883 & 7.825 & 10.851 \\
\hline & Hall & 100188750 & 12.089 & 6.517 & 8.654 & 13.938 \\
\hline & Coastguard & 116643040 & 2.720 & 9.193 & 11.055 & 13.222 \\
\hline & Silent & 108745500 & 5.140 & 4.778 & 6.674 & 7.115 \\
\hline & Waterfall & 135369020 & 14.294 & 7.740 & 7.542 & 14.253 \\
\hline & Average & 112561114 & 7.954 & 6.822 & 8.350 & 11.876 \\
\hline \multirow{6}{*}{$\begin{array}{l}\text { HDTV } \\
(720 p)\end{array}$} & Parkrun & 1255203920 & 5.724 & 4.863 & 7.095 & 11.015 \\
\hline & Shields & 1092507520 & 9.025 & 4.437 & 6.591 & 12.388 \\
\hline & Bigships & 886159200 & 4.871 & 8.331 & 10.922 & 12.388 \\
\hline & City & 936586320 & 4.952 & 10.123 & 14.626 & 19.091 \\
\hline & Crew & 725805600 & 4.910 & 7.207 & 10.744 & 13.912 \\
\hline & Average & 979252512 & 5.896 & 6.992 & 9.996 & 13.759 \\
\hline
\end{tabular}

\section{CONCLUSION}

In this paper, a hierarchical intra prediction algorithm making use of an adaptive interpolation filter is proposed to improve lossless intra compression process. Based on the hierarchical prediction, some pixels in a MB/block are firstly predicted and encoded with the minimal bitrate, and then others are predicted based on an adaptive interpolation filter by using the nearest reference pixels. The hierarchical prediction makes the intra prediction more accurate due to the use of adaptive interpolation and adjacent samples as references, and thus the information left in the residual block can be reduced significantly. Two coding modes are then used to efficiently encode the residual block by using the rate optimization method. Experimental results show that the proposed algorithm can improve the compression efficiency of the lossless coding in the H.264/AVC standard when compared with other algorithms in the literature.

Acknowledgment: This work is supported by the Centre for Multimedia Signal Processing, The Hong Kong Polytechnic University (G-U863).

\section{REFERENCES}

[1] M.J. Weinberger, "The LOCO-I lossless image compression algorithm: principles and standardization into JPEG-LS," IEEE Transaction on Image Processing, vol.9, no.8, pp.1309-1324, Aug. 2000.

[2] Motion JPEG2000 Final Committee Draft, ISO/IEC JTC 1/SC29/WG1, Document N2117, Mar. 2001.

[3] G.J. Sullivan, P. Topiwala, and A. Luthra, "The H.264/AVC Advanced Video Coding Standard: Overview and Introduction to the Fidelity Range Extensions". Retrieved Oct. 2004.

[4] Y.L. Lee, K.H. Han and G.J. Sullivan, "Improved lossless intra coding for H.264/MPEG-4 AVC," IEEE Transaction on Image Processing, vol.15, pp.2610-2615, September 2006.

[5] S.T. Wei, S.R. Shen, B.D. Liu and J.F. Yang, "Lossless image and video coding based on H.264/AVC intra predictions with simplified interpolations”, Proceeding, ICIP'2009, pp.633-636, Nov. 2009, Cairo Egypt.

[6] L.L. Wang and W.C. Siu, "Improved Lossless Coding Algorithm in H.264/AVC based on Hierarchical Intra Prediction and Coding Mode Selection", Journal of Electronic Imaging 20(4), 043001 (Oct.-Dec. 2011).

[7] Y. Vatis and J. Ostermann, "Adaptive Interpolation Filter for H.264/AVC," IEEE Transactions on Circuits and Systems for Video Technology, Vol.19, NO.2, pp.179-192, Feb. 2009

[8] X. Li and M.T. Orchard, "New Edge-Directed Interpolation," IEEE Transactions on Image Processing, vol.10, no.10, pp.1521-1527, Oct. 2001.

[9] JVT H.264/AVC Reference Software version JM 18.0, http://iphome.hhi.de/suehring/tml/download/old_jm/

[10] G. Sullivan, "Recommended Simulation Common Conditions for Coding Efficiency Experiments Revision 1", 31st VCEGAE010R1 meeting, Marrakech, Morocco, 13-19 January, 2007.

[11] http://www.vcodex.com/h265.html

[12] K.Y. Liao, J.F. Yang and M.T. Sun, "Rate-Distortion Cost Estimation for H.264/AVC," IEEE Transactions on Circuits and Systems for Video Technology Vol.20, NO.1, pp.38-49, Jan. 2010 . 